\title{
Aerobic Glycolysis Is Required for Spatial Memory Acquisition But Not Memory Retrieval in Mice
}

\author{
Richard A. Harris, ${ }^{1 *}$ Asad Lone, ${ }^{1 *}$ Heeseung Lim, ${ }^{2}$ Francisco Martinez, ${ }^{3}$ (D)Ariel K. Frame, ${ }^{1}$ (1)Timothy J. \\ Scholl, ${ }^{2,3,4}$ and ${ }^{\circledR}$ Robert C. Cumming ${ }^{1}$
}

https://doi.org/10.1523/ENEURO.0389-18.2019

${ }^{1}$ Department of Biology, University of Western Ontario, London, Ontario N6A 5B7, Canada, ${ }^{2}$ Department of Medical Biophysics, University of Western Ontario, London, Ontario N6A 5C1, Canada, ${ }^{3}$ Robarts Research Institute, University of Western Ontario, London, Ontario N6A 3K7, Canada, ${ }^{4}$ Ontario Institute for Cancer Research, Toronto, Ontario M5G OA3, Canada

\begin{abstract}
The consolidation of newly formed memories and their retrieval are energetically demanding processes. Aerobic glycolysis (AG), also known as the Warburg effect, consists of the production of lactate from glucose in the presence of oxygen. The astrocyte neuron lactate shuttle hypothesis posits that astrocytes process glucose by AG to generate lactate, which is used as a fuel source within neurons to maintain synaptic activity. Studies in mice have demonstrated that lactate transport between astrocytes and neurons is required for long-term memory formation, yet the role of lactate production in memory acquisition and retrieval has not previously been explored. Here, we examined the effect of dichloroacetate (DCA), a chemical inhibitor of lactate production, on spatial learning and memory in mice using the Morris water maze (MWM). In vivo hyperpolarized ${ }^{13} \mathrm{C}$-pyruvate magnetic resonance spectroscopy revealed decreased conversion of pyruvate to lactate in the mouse brain following DCA administration, concomitant with a reduction in the phosphorylation of pyruvate dehydrogenase. DCA exposure before each training session in the MWM impaired learning, which subsequently resulted in impaired memory during the probe trial. In contrast, mice that underwent training without DCA exposure, but received a single DCA injection before the probe trial exhibited normal memory. Our findings indicate that AG plays a key role during memory acquisition but is less important for the retrieval of established memories. Thus, the activation of AG may be important for learning-dependent synaptic plasticity rather than the activation of signaling cascades required for memory retrieval.
\end{abstract}

Key words: aerobic glycolysis; lactate; magnetic resonance spectroscopy; memory; metabolism; synaptic plasticity

\section{Significance Statement}

Neuronal activation is an energetically demanding process. The brain is mainly fueled by glucose, yet a substantial portion of this metabolite is converted to lactate despite the presence of adequate oxygen, a phenomenon known as aerobic glycolysis (AG). The transport of lactate between astrocytes and neurons is key for learning and memory, yet the role of lactate production in these processes is poorly understood. Here we report that the administration of dichloroacetate (DCA), a chemical inhibitor of AG, attenuates the conversion of pyruvate to lactate in the brains of mice. DCA exposure impaired spatial learning but had no effect on the retrieval of an established memory. These observations suggest that lactate production may be required for memory acquisition but not retrieval. 


\section{Introduction}

Glucose is the primary fuel source within the human brain (Mergenthaler et al., 2013) and can be processed by various metabolic pathways to produce energy. Under normal oxygen tension, glucose is typically broken down in the cytosol to pyruvate, which then enters the mitochondria and undergoes oxidative decarboxylation by pyruvate dehydrogenase $(\mathrm{PDH})$ to produce acetyl-CoA. Acetyl-CoA provides carbon atoms for the tricarboxylic acid cycle leading to the production of $\mathrm{NADH}$ and $\mathrm{FADH}_{2}$, ultimately generating 32-36 ATP molecules by oxidative phosphorylation. Under hypoxic conditions, pyruvate is preferentially converted to lactate by lactate dehydrogenase (LDHA) to produce two ATP molecules in a process known as anaerobic glycolysis. However, a third way to process glucose, termed aerobic glycolysis (AG), occurs when glucose is processed to generate lactate despite normal oxygen tension. Under resting conditions, AG accounts for $10-15 \%$ of glucose consumed in the human brain and increases to up to $40 \%$ following neuronal activation (Raichle et al., 1970; Boyle et al., 1994; Madsen et al., 1998, 1999; Powers et al., 2007). Recent evidence has implicated lactate, the end product of $A G$, in the regulation of synaptic plasticity and gene expression changes associated with learning and memory (Suzuki et al., 2011; Goyal et al., 2014; Yang et al., 2014; Shannon et al., 2016). Astrocytes are believed to be the primary cell type in the CNS that uses AG to generate lactate, which is subsequently transported to neurons to meet their high energy needs (Magistretti and Allaman, 2018). Astrocytic glycogen can also be mobilized via glycogenolysis to generate glucose for subsequent processing by $A G$ to generate lactate (Brown et al., 2004). Numerous studies in rodents have shown that the inhibition of astrocytic glycogenolysis or lactate transport results in compromised learning and memory (Magistretti and Allaman, 2018). However, the effect of AG inhibition on cognitive processes has yet to be examined.

Dichloroacetate (DCA) is a blood-brain barrier (BBB)permeable chemical that selectively inhibits $\mathrm{PDH}$ kinase (PDK; Kato et al., 2008). Phosphorylation of PDH by PDK results in a strong suppression of $\mathrm{PDH}$ activity, thereby favoring AG and lactate production (Kato et al., 2008).

Received October 3, 2018; accepted January 26, 2019; First published February 11, 2019.

The authors declare no competing financial interests.

R.A.H., T.J.S., and R.C.C. designed research; R.A.H., A.L., H.L., F.M., and A.K.F. performed research; R.A.H., A.L., and R.C.C. analyzed data; R.A.H., A.L., and R.C.C. wrote the paper.

This research was supported by Natural Sciences and Engineering Research Council of Canada Grants 355803-2013 (R.C.C.) and 06338-2017 (T.J.S.), Scottish Rite Charitable Foundation Grant 11103 (R.C.C.), Canada Foundation for Innovation Grant 22167 (R.C.C.), and Canadian Consortium on Neurodegeneration in Aging Grant 137794 (R.C.C.).

${ }^{\star}$ R.A.H. and A.L. contributed equally to this work.

Correspondence should be addressed to Robert C. Cumming at rcummin5@uwo.ca.

https://doi.org/10.1523/ENEURO.0389-18.2019

Copyright (C) 2019 Harris et al.

This is an open-access article distributed under the terms of the Creative Commons Attribution 4.0 International license, which permits unrestricted use, distribution and reproduction in any medium provided that the original work is properly attributed.
Based on its ability to inhibit PDK and promote optimal mitochondrial PDH activity, DCA has been used clinically to lower lactate levels in the blood and CSF of patients with congenital lactic acidosis (Stacpoole et al., 2003). In this study, we examined the effect of DCA-mediated inhibition of AG on spatial learning and memory in mice. Exposure to DCA readily prevented the phosphorylation of $\mathrm{PDH}$ and reduced the conversion of pyruvate to lactate within the brain. Moreover, DCA administration significantly impaired spatial learning but had no effect on the recall of established memories, suggesting that $A G$ may play a role in memory acquisition but not retrieval.

\section{Materials and Methods}

\section{Animals}

Male C57BL/6J mice (RRID:IMSR_JAX:000664) were housed in groups under a $12 \mathrm{~h}$ light/dark cycle with ad libitum access to base chow (2018 Teklad Global Diet, Envigo) and tested at 9 months of age. All animal procedures were performed in compliance with the Canadian Council on Animal Care guidelines under an animal protocol approved by the University of Western Ontario animal care committee.

\section{Hyperpolarized 13C-pyruvate magnetic resonance spectroscopy}

Images were acquired using a multinuclear-capable 3.0 Tesla MRI system (Discovery MR750 3.0 T; GE Healthcare). A custom-built dual-tuned ${ }^{1} \mathrm{H}-{ }^{13} \mathrm{C}$ solenoid radio frequency coil was used to facilitate inherent registration of ${ }^{1} \mathrm{H}$ and ${ }^{13} \mathrm{C}$ images. A buffered hyperpolarized [1${ }^{13} \mathrm{C}$ ]pyruvate (95\% enriched ${ }^{13} \mathrm{C}$ content; Sigma-Aldrich) solution was produced using dynamic nuclear polarization (HyperSense, Oxford Instruments). The hyperpolarized solution had a final concentration of $150 \mathrm{~mm}$ pyruvate with $\mathrm{pH} 7.4$ at $37^{\circ} \mathrm{C}$ and $\sim 10 \%$ polarization producing a signal enhancement factor of $>10,000$ for magnetic resonance spectroscopy imaging (MRSI). The in vivo spinlattice relaxation time was $\sim 45 \mathrm{~s}$. The imaging session consisted of fast imaging using steady-state freeprecession ${ }^{1} \mathrm{H}$ image acquisition (FIESTA) and hyperpolarized ${ }^{13} \mathrm{C}$ MRSI. Before ${ }^{13} \mathrm{C}$ MRSI, FIESTA images were acquired with the following imaging parameters: $30 \times 30$ $\mathrm{mm}$ field of view (FOV), $0.2 \mathrm{~mm}$ isotropic in-plane resolution, $0.4 \mathrm{~mm}$ slice thickness, repetition time $(T R)=10.3$ $\mathrm{ms}$, echo time $=5.2 \mathrm{~ms}$, bandwidth $=12.58 \mathrm{~Hz}$, and phase cycling $=8$. For hyperpolarized ${ }^{13} \mathrm{C} \mathrm{MRSI}$, a $\sim 0.3$ $\mathrm{ml}$ bolus of the hyperpolarized $\left[1-{ }^{13} \mathrm{C}\right]$ pyruvate-buffered solution was injected over $10 \mathrm{~s}$ via a tail vein catheter and allowed to circulate for $15 \mathrm{~s}$ for cell uptake and conversion of the ${ }^{13} \mathrm{C}$-labeled pyruvate before imaging. The 2D MRSI was performed using a free induction decay chemical shift pulse sequence with the following parameters: $30 \times 30$ $\mathrm{mm}$ FOV, $2.5 \mathrm{~mm}$ isotropic in-plane resolution, slice thickness $=10 \sim 15 \mathrm{~mm}$, TR $=80 \mathrm{~ms}$, spectral width $=5000$ $\mathrm{Hz}$, and number of points $=256$. Total MRSI acquisition time was $\sim 12 \mathrm{~s}$. DCA (Sigma-Aldrich) was freshly prepared at a concentration of $40 \mathrm{mg} / \mathrm{ml}$ in sterile saline and neutralized to $\mathrm{pH} 7.4 \pm 0.1$ using $\mathrm{NaOH}$. Mice were given $30 \mathrm{~min}$ to recover following hyperpolarized ${ }^{13} \mathrm{C}$-pyruvate 
injection before injection of DCA at $200 \mathrm{mg} / \mathrm{kg}$ via tail vein catheter. Mice were then given an additional $30 \mathrm{~min}$ to recover, followed by another bolus of hyperpolarized ${ }^{13} \mathrm{C}$ pyruvate via a tail vein catheter. Mice immediately underwent a second ${ }^{13} \mathrm{C}$ MRSI session, as previously described. Intravenous injection of DCA, as opposed to intraperitoneal injection used in learning and memory testing (described below), was necessary due to the technical requirement of maintaining exact head position during all stages of MRSI.

\section{Western blot analysis of brain extracts}

Mice were sedated in a $\mathrm{CO}_{2}$ chamber and then immediately perfused with Dulbecco's PBS, pH 7.4 containing $2 \mathrm{~mm}$ leupeptin, $0.1 \mathrm{~mm}$ pepstatin A, $100 \mathrm{~mm}$ EDTA, $1 \mathrm{~mm}$ PMSF, and $0.5 \mathrm{~mm}$ sodium orthovanadate. The brain was removed, and the frontal cortex of the right hemisphere was homogenized in an extraction buffer containing 50 $\mathrm{mm}$ Tris $\mathrm{pH} 7.5,2 \%$ SDS, and protease and phosphatase inhibitors. Protein extracts were resolved by $10 \%$ SDSPAGE, and electroblotted onto a PVDF membrane (BioRad). Membranes were probed with the following antibodies: PDK1 (catalog \#KAP-PK112D, Enzo Life Sciences; RRID:AB_1193509), PDH-E1 $\alpha$ (pSer $^{232}$; catalog \#AP1063, Millipore; RRID:AB 10616070), PDH-E1 $\alpha$ (catalog \#ab110330, Abcam; RRID:AB_10858459), and $\beta$-actin (catalog \#3700, Cell Signaling Technology; RRID: AB_2242334). Bands were detected using Luminata Forte chemiluminescence substrate (EMD Millipore) and imaged using a Chemidoc XRS System (Bio-Rad). Densitometric analysis was performed using Quantity One 1-D Analysis Software (Bio-Rad; RRID:SCR_014280).

\section{Water maze apparatus}

The Morris water maze (MWM) consisted of a uniformly white circular pool with a diameter of 48 inches and a height of 30 inches (San Diego Instruments) and was divided into four fictive quadrants. The pool was filled with water and maintained at a temperature of $24^{\circ} \mathrm{C}$ using a $300 \mathrm{~W}$ submersible aquarium heater (Aqueon). Spatial visual cues in the form of different-shaped cardboard pictures were placed on the walls surrounding the water tank. Mice were first submitted to a single habituation session of three trials, in which the mouse was placed on circular plastic platform (diameter, $10.16 \mathrm{~cm}$ ) positioned 1 $\mathrm{cm}$ below the surface of the water in the center of the pool for $15 \mathrm{~s}$. Following habituation, mice were trained for 4 consecutive days, with four trials per day to find the location of the circular platform located in the center of the target quadrant. The release point for the first trial of the day corresponded to the cardinal directions (i.e., North, East, South, West), rotating clockwise with each new training day. For each subsequent trial session on a given day, mice were released into the water at a position that was a one-quarter turn clockwise from the previous trial release point. A trial lasted either until the mouse found the platform or $90 \mathrm{~s}$ had elapsed, at which point the mouse was gently guided to the platform and left there for $15 \mathrm{~s}$ before it was removed. Following each trial, mice were toweled dry and placed in their home cage for an intertrial interval of 10 min to allow recovery and ensure the consistency of timing between trials. On the fifth day, the platform was removed, and the mouse was released in the center of the tank and given $60 \mathrm{~s}$ to attempt to find the missing platform. A second probe trial was performed 1 week later, using the same procedure as the first probe trial. A flag trial was also performed in which the platform was placed back into the water within the quadrant that was opposite to that used during the training trials, and a small red plastic pole was inserted into the platform so as to create a visual cue. The mouse was released in the center of the tank and given $90 \mathrm{~s}$ to find the location of the platform. Visual data were collected and analyzed using ANY-maze version 4.98 (ANY-maze; RRID:SCR_014289). Animals were injected intraperitoneally with either saline or DCA (Sigma-Aldrich) at a dose of $200 \mathrm{mg} / \mathrm{kg}$. One cohort of mice was injected with either saline or DCA 30 min before the start of each training session, while a second cohort of mice underwent normal training but received either a saline or DCA injection $30 \mathrm{~min}$ before the probe trial.

\section{Statistical analysis}

A Welch's paired $t$ test was used to analyze the difference between the ratio of lactate to pyruvate for before and after DCA injection. A Welch's unpaired $t$ test was used to analyze band densitometry and memory performance for saline- and DCA-injected mice. A two-way ANOVA with repeated measures was used to analyze the differences among latency to the platform, path length, and mean speed for saline- and DCA-injected mice during training. Statistical evaluation was performed using RStudio version 0.97.551 (RStudio; RRID:SCR_000432).

\section{Results}

\section{DCA reduces conversion of pyruvate to lactate in the mouse brain}

To test whether DCA was a suitable compound for manipulating $A G$ and lactate production in the brain, mice were analyzed using hyperpolarized ${ }^{13} \mathrm{C}$-pyruvate magnetic resonance spectroscopy $30 \mathrm{~min}$ before, and $30 \mathrm{~min}$ after, the injection of DCA $(200 \mathrm{mg} / \mathrm{kg})$ via an intravenous tail-vein catheter (Fig. $1 A$ ). ${ }^{13} \mathrm{C}$-pyruvate spectra were collected within individual voxels throughout the whole brain (Fig. 1B) and combined to calculate the ratio of lactate to pyruvate as an indication of pyruvate conversion to lactate in the mouse brain before and after DCA injection (Fig. 1C). The observed lactate-to-pyruvate metabolite ratio within the brain was significantly reduced from $0.144 \pm 0.034$ (mean \pm SEM) before DCA exposure to $0.056 \pm 0.016$ after DCA administration $\left(t_{(1,10)}=2.753\right.$, $p<0.05$; Fig. $1 D$ ). Thus, DCA administration reduces lactate production from pyruvate in the mouse brain 30 min after infusion, indicating that it is a suitable compound for inhibiting AG.

\section{DCA injection reduces the phosphorylation of PDH in the frontal cortex and hippocampus}

To confirm that DCA exerts an effect on the brain regions related to spatial learning and memory, the phosphorylated form of PDH was measured in brain extracts 
A

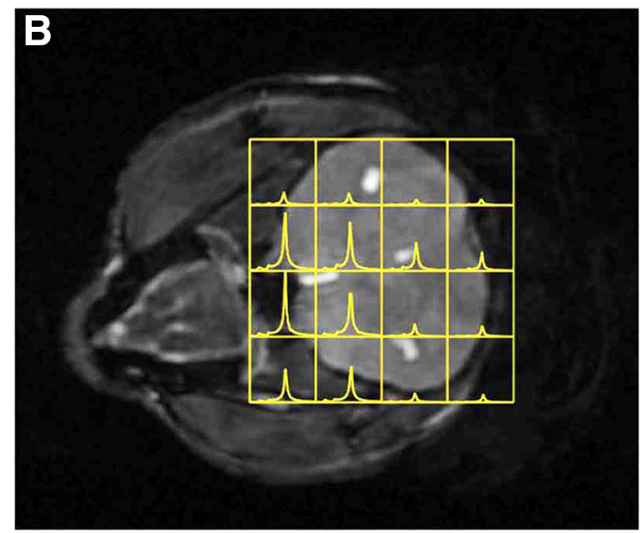

C

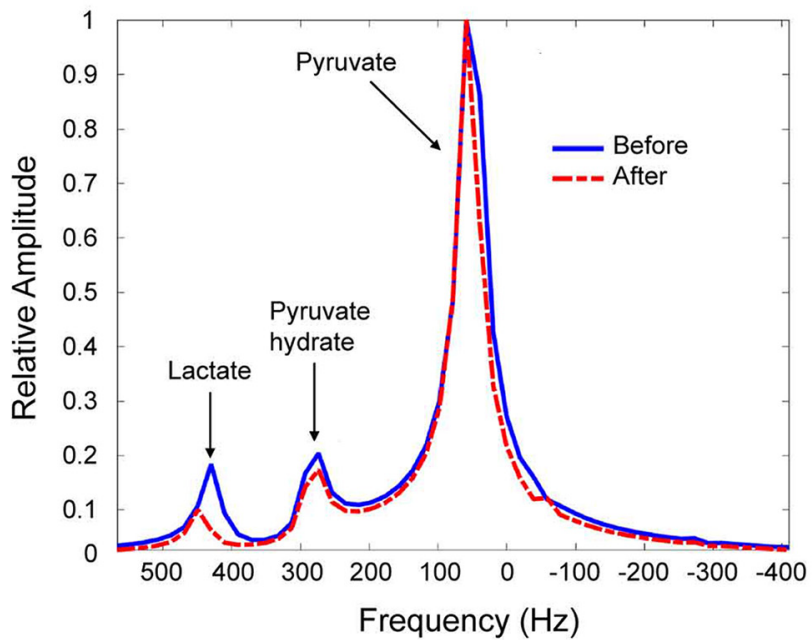

D

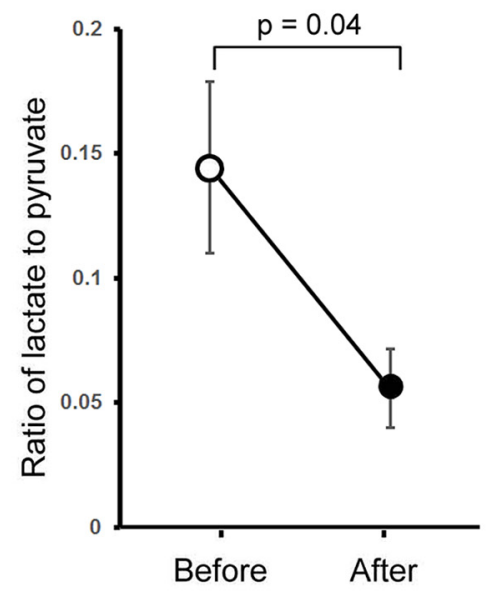

Figure 1. Hyperpolarized ${ }^{13} \mathrm{C}$-pyruvate magnetic resonance spectroscopic imaging reveals a decline in lactate following DCA administration. $\boldsymbol{A}$, Injection and imaging regime of 9 -month-old mice using hyperpolarized MRSI of [1- $\left.{ }^{13} \mathrm{C}\right]$ pyruvate to measure the conversion of pyruvate to lactate. Mice were imaged after the first ${ }^{13} \mathrm{C}$-pyruvate injection (Before) and 30 min later were then injected with DCA (200 mg/kg). Following a $30 \mathrm{~min}$ recovery time, another injection of ${ }^{13} \mathrm{C}$-pyruvate and imaging were performed (After). $\boldsymbol{B}$, ${ }^{1} \mathrm{H} \mathrm{MRI}$ of the brain in the coronal field overlayed with MRSI voxels containing spectra of ${ }^{13} \mathrm{C}$-labeled pyruvate and lactate (yellow). $\boldsymbol{C}$, Conversion of pyruvate to lactate was measured as a ratio of the observed lactate peak to pyruvate peak from before DCA injection (blue line) and after (red dashed line). A pyruvate hydrate peak was also recorded. $\boldsymbol{D}$, DCA injection reduces the ratio of lactate to pyruvate in the mouse brain $(p=0.04)$. Data shown are the mean \pm SEM. $n=4$.

from the frontal cortex and hippocampus 30 min after intraperitoneal injection of DCA. DCA administration caused a significant decrease in PDH phosphorylation levels in both the frontal cortex $\left(t_{(1,10)}=13.78, p<0.001\right)$ and hippocampus $\left(t_{(1,14)}=4.493, p<0.001\right)$ compared with saline-injected mice (Fig. 2). A significant decrease in PDK1 expression was also observed in the frontal cortex of DCA-injected mice $\left(t_{(1,10)}=2.837, p<0.05\right)$. These results are in accordance with previous observations that DCA readily crosses the blood-brain barrier and targets AG by increasing PDH activity (Kato et al., 2008).

\section{DCA administration causes impairment in spatial learning}

To determine whether $A G$ is required for spatial learning, a task that is dependent on communication between the hippocampus and frontal cortex, mice were injected intraperitoneally with DCA 30 min before each training session for the MWM task. Several measures were re- corded for each training day, including the latency to find the platform, the total path length, and the mean speed (Fig. $3 A-C$ ). DCA administration resulted in a significant reduction in learning, as reflected by the increased latency time to find the platform $\left(F_{(1,12)}=9.83, p<0.01\right)$ and increased path length $\left(F_{(1,12)}=12.84, p<0.01\right)$, compared with saline-injected mice. There was no significant difference in mean speed between the two groups $\left(F_{(1,12)}\right.$ $=2.93, p=0.11$ ). Mice exposed to DCA during training subsequently exhibited a reduced ability to locate the platform during the probe trial, as visualized by a trace analysis of the swim path in a heat map (Fig. 3D). DCAinjected mice also displayed a significantly reduced percentage of time spent in the correct quadrant $\left(t_{(1,12)}=\right.$ $2.21, p<0.05)$ and a reduction in the number of platform entries $\left(t_{(1,12)}=3.71, p<0.05\right)$ compared with salineinjected mice, yet they displayed no difference in total distance covered during the trial $\left(t_{(1,12)}=0.01, p=0.99\right.$; Fig. $3 E-G)$. A second probe trial, performed 1 week later, 
A

Frontal Cortex
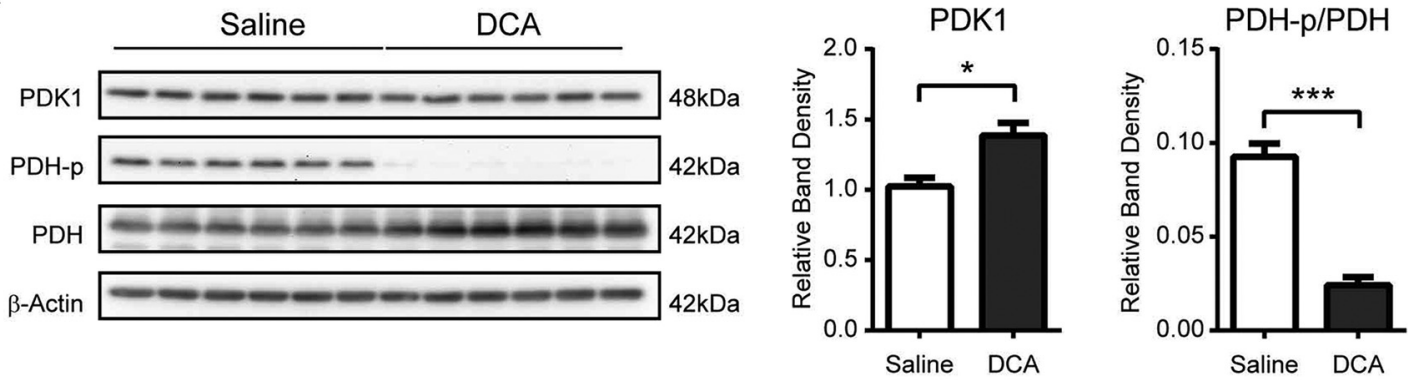

B

Hippocampus
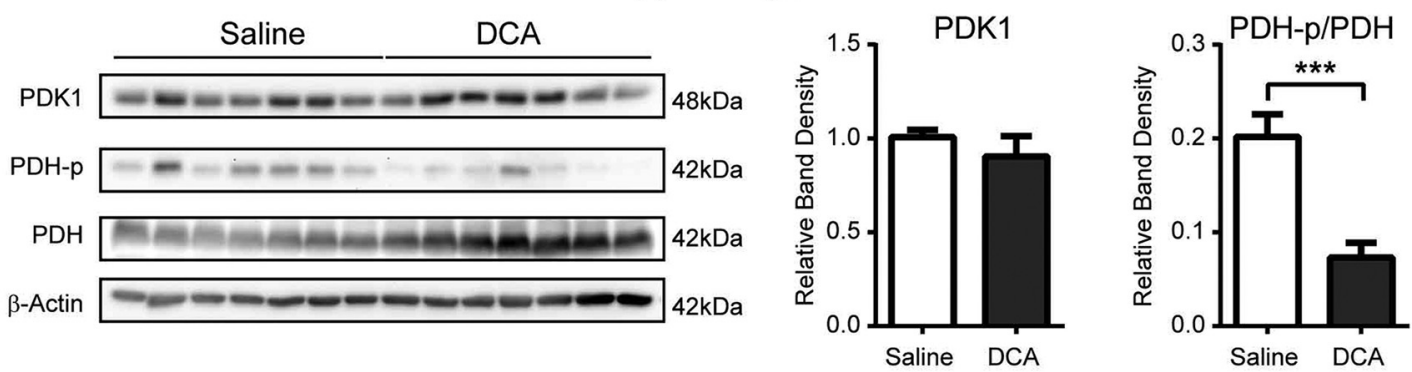

Figure 2. DCA injection reduces the phosphorylation of $\mathrm{PDH}$ in the frontal cortex and hippocampus. $\boldsymbol{A}, \boldsymbol{B}$, Western blot analysis (left) was performed on extracts from the frontal cortex $(\boldsymbol{A})$ and the hippocampus $(\boldsymbol{B})$ of mice intraperitoneally injected with either saline or DCA $(200 \mathrm{mg} / \mathrm{kg}) 30 \mathrm{~min}$ before being killed. Densitometric analysis of Western blots (right) revealed significantly lower PDH phosphorylation in DCA-treated mice relative to saline-injected mice $(* p<0.05, * * * p<0.001 ; n=6$ and 6 , respectively, for extracts from the frontal cortex of saline- and DCA-injected mice; and $n=7$ and 7 , respectively, for extracts from the hippocampus of salineand DCA-injected mice). Band densities were standardized to $\beta$-actin controls. Data shown are the mean \pm SEM.

revealed that DCA exposure during training impaired long-term memory, as indicated by a significant decrease in number of platform crosses $\left(t_{(1,12)}=3.25, p<0.01\right)$ compared with saline-treated controls (Fig. $3 H$ ). Thus, DCA injection caused an impairment in learning that subsequently weakened memory association with the location of the platform both $24 \mathrm{~h}$ and $7 \mathrm{~d}$ after training.

\section{DCA administration does not interfere with memory recall}

We next asked whether $A G$ is required for spatial memory recall. Mice underwent $4 \mathrm{~d}$ of training in the MWM and were then injected intraperitoneally with DCA 30 min before the probe trial on the fifth day (Fig. 4A). DCA-injected mice showed no difference in memory performance from saline-injected mice, as demonstrated visually by a trace analysis of the swim path in a heat map (Fig. 4B). The total distance covered in the probe trial revealed no significant difference between saline- and DCA-injected mice $\left(t_{(1,16)}\right.$ $=0.80, p=0.43$; Fig. $4 C$ ). In addition, no significant differences in memory performance was observed, as indicated by the percentage of time spent in the correct quadrant $\left(t_{(1,16)}=0.52, p=0.31\right)$ and the total number of platform entries $\left(t_{(1,16)}=0.77, p=0.45\right.$; Fig. $\left.4 D, E\right)$. To confirm that DCA had no adverse effect on vision, a flag trial was performed that demonstrated that saline- and DCA-injected mice exhibited similar times to find the flag $\left(t_{(1,16)}=0.16, p=0.44\right.$; Fig. $\left.4 F\right)$. These findings indicate that $A G$ is not explicitly required for spatial memory re- trieval. In addition, DCA injection had no adverse effects on swimming performance or visual acuity.

\section{Discussion}

The role of glycogenolysis and lactate transport in learning and memory has been extensively studied (Magistretti and Allaman, 2018). Rats undergoing training during an inhibitory avoidance (IA) test exhibit a transient elevation of hippocampal lactate levels, measured by in vivo microdialysis, that is completely abolished by bilateral injection of the glycogenolysis inhibitor 1,4-dideoxy1,4-imino-D-arabinitol (DAB) into the hippocampus (Suzuki et al., 2011). In a separate study, DAB injection in the rat hippocampus 5 min before behavioral testing significantly impaired spatial working memory in a four-arm spontaneous alternation task (Newman et al., 2017). Moreover, DAB-mediated impairment of spatial working memory was rescued by hippocampal injection of lactate (Newman et al., 2011). Chemical or genetic inhibition of monocarboxylate transporters that shuttle lactate in the brain also impairs IA and spatial working memory (Newman et al., 2011; Suzuki et al., 2011). Lactate derived from astrocytic glycogenolysis, and its subsequent transport to neurons, was shown to mediate hippocampal long-term potentiation (LTP) and to induce learning-dependent molecular changes, including increased expression of immediate early genes such as activity-regulated cytoskeletal protein (Arc), proto-oncogene c-Fos, early growth response 1 (Egr1), and phosphorylation of cAMP response 
A

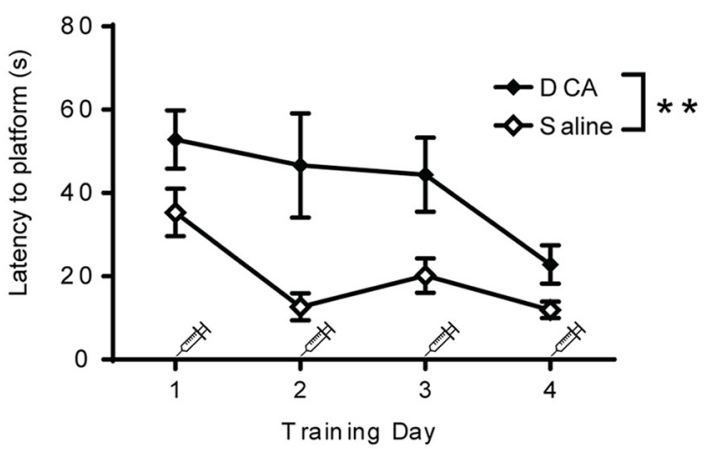

B

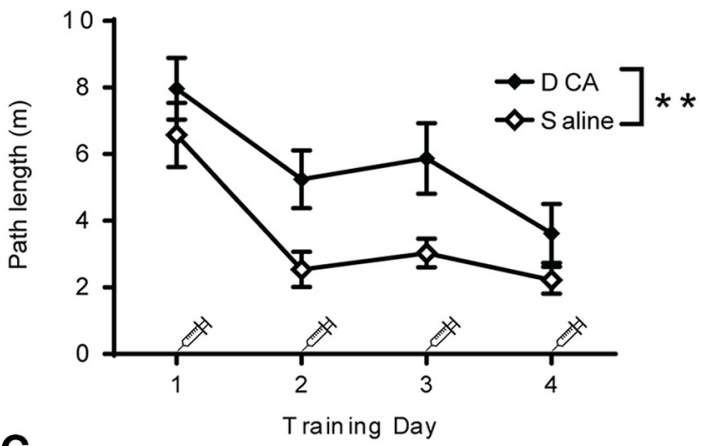

C

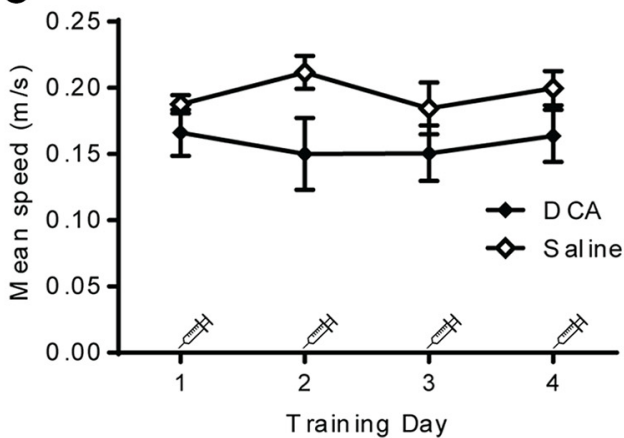

D

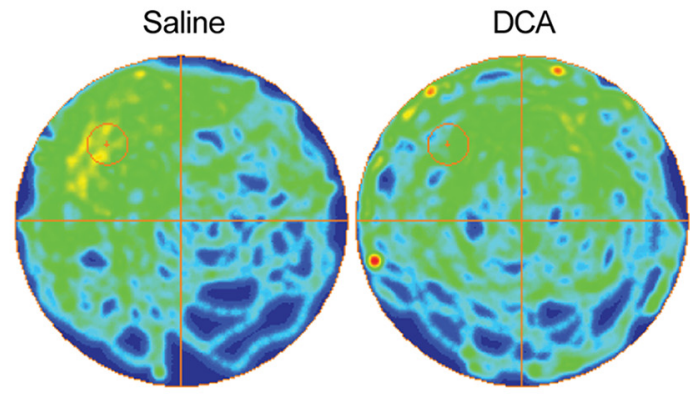

E

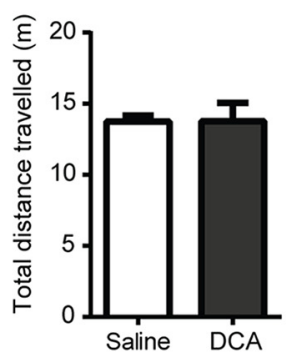

F

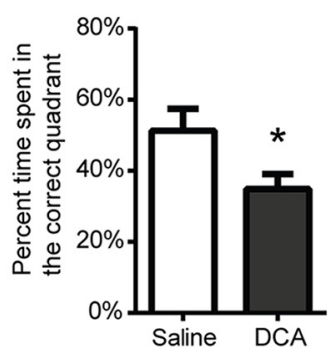

H

G

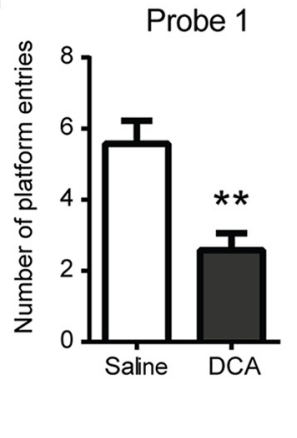

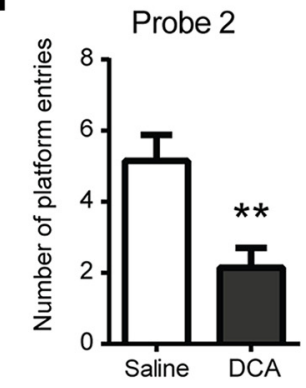

Figure 3. DCA administration causes impairment in spatial learning. Mice were intraperitoneally injected with saline or DCA (200 $\mathrm{mg} / \mathrm{kg}$ ) $30 \mathrm{~min}$ before each day of training and allowed to find the location of a hidden platform in the northwest quadrant. $\boldsymbol{A}-\boldsymbol{C}$, The latency to find the platform $(\boldsymbol{A})$, the total path length $(\boldsymbol{B})$, and the mean speed $(\boldsymbol{C})$ were recorded on each training day. $\boldsymbol{D}-\mathbf{G}$, On day 5 , a probe trial was performed without DCA injection, and mice were allowed to swim for $60 \mathrm{~s}$. $\boldsymbol{D}$, The swim path for each group of mice was compiled into heat map representations. $\boldsymbol{E}-\boldsymbol{G}$, Measurements were taken for the total distance traveled $(\boldsymbol{E})$, the percentage of time spent in the correct quadrant $(\boldsymbol{F})$, and the number of times the boundary of the platform was crossed $(\boldsymbol{G})$. $\boldsymbol{H}$, A week after the first probe trial, mice were again tested with a second probe trial, and measurements were taken for the number of times the boundary of the platform was crossed. Data shown are the mean \pm SEM. $* p<0.05, * * p<0.01 . n=7$ and 7 for saline and DCA treatments, respectively.

element-binding protein and cofilin (Suzuki et al., 2011; Yang et al., 2014). Collectively, these observations provide strong support for the astrocyte neuron lactate shuttle model in which glucose, either directly or supplied via astrocytic glycogenolysis, is processed by AG within astrocytes to generate lactate, which is then transported to neurons to fuel the high energy demands associated with the cellular and molecular changes underlying memory formation and storage. However, the precise role of taskbased activation of AG in memory acquisition and retrieval is poorly defined.

Several studies have demonstrated a task-associated decrease in extracellular glucose and a concurrent rise in extracellular lactate in the hippocampus during spatial memory processing (McNay et al., 2006, 2000; Newman et al., 2017). In addition, genes involved in AG, including LDHA and PDK, show increased hippocampal expression following IA learning, suggesting that the induction of enzymes that promote lactate production may be required for memory acquisition (Tadi et al., 2015). Rats also exhibit increased $A G$ in the amygdala during IA training and memory testing (Sandusky et al., 2013). Interestingly, a marked increase in extracellular pyruvate was observed in the amygdala during IA training, whereas a significant drop in pyruvate was observed during memory testing (Sandusky et al., 2013). These observations prompted the 
A

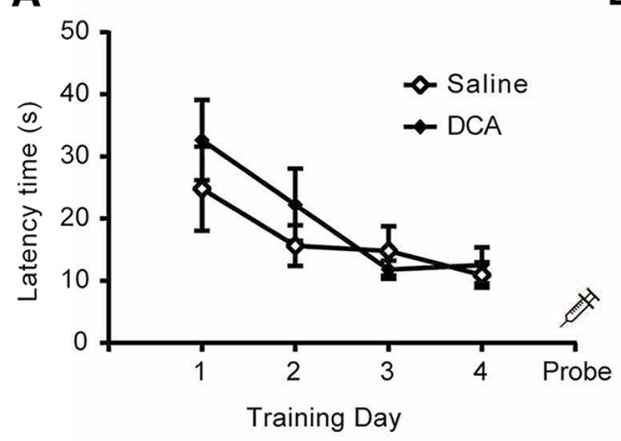

B

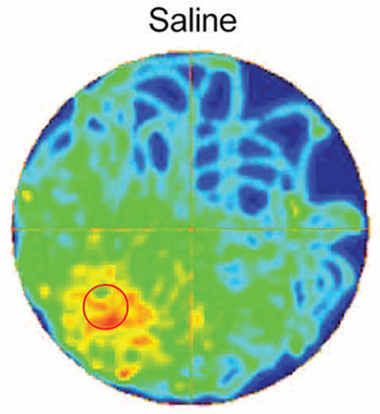

E

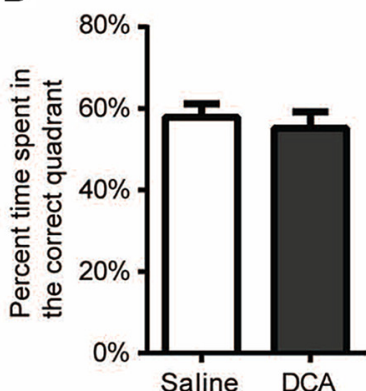

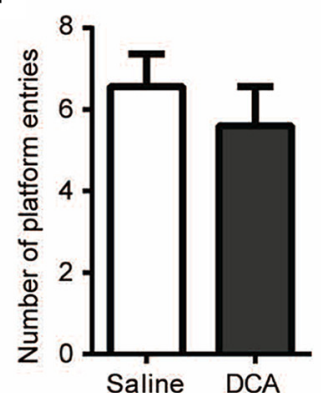

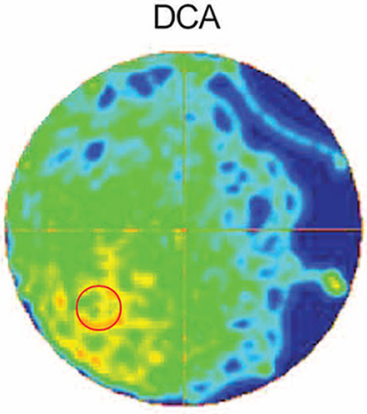

F

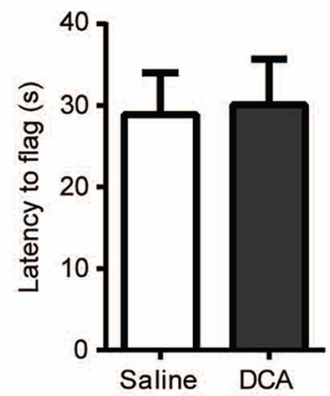

Figure 4. DCA administration does not interfere with memory retrieval. MWM performed by mice intraperitoneally injected with saline or DCA (200 mg/kg) on the probe trial. A, Mice were trained for 4 consecutive days ( 4 trials $/ \mathrm{d})$ to find the location of a hidden platform in the southwest quadrant, and the latency to escape was recorded. $\mathbf{B}-\mathbf{G}$, On day 5, a probe trial was performed in which the platform was removed and mice were allowed to swim for $60 \mathrm{~s}$. B. The swim path for each group of mice was recorded and compiled into heat map representations. $\boldsymbol{C}-\boldsymbol{E}$, Measurements were taken for the total distance traveled $(\boldsymbol{C})$, the percentage of time spent in the correct quadrant $(\boldsymbol{D})$, and the number of times the boundary of the platform was crossed $(\boldsymbol{E})$. $\boldsymbol{F}$, Immediately after the probe trial, a flag trial was performed and the latency to find the flag was recorded. Data shown are the mean \pm SEM. $n=9$ and 10 for saline and DCA treatments, respectively. No significant differences were observed between saline-treated (sham) and DCA-injected mice.

authors to speculate that memory acquisition relies predominately on AG, whereas memory retrieval places a higher demand on oxidative metabolism. Our findings are in accordance with this theory, as discussed below.

In this study, we demonstrate for the first time that systemic DCA administration causes a decline in lactate production from pyruvate with a concomitant reduction in $\mathrm{PDH}$ phosphorylation in the frontal cortex and hippocampus, brain regions that are critical for spatial memory formation. In support of previous studies, we also show evidence that DCA can readily cross the BBB and inhibit PDK activity, thereby lowering PDH phosphorylation and reducing lactate production (Abemayor et al., 1984; Stacpoole et al., 2003). DCA injection before each training trial resulted in impaired learning, as reflected by the increased latency and increased total path length required to find the platform in the MWM. In addition, DCAmediated inhibition of AG during training resulted in a reduced ability to locate the platform during subsequent probe trials. Although DCA exposure may potentially cause nonspecific effects (disorientation, and perceptual and motivational alterations), this is unlikely because we did not detect a significant difference in the mean speed during training or a decrease in the ability to find the flag during the probe trial following DCA injection. Interestingly, a single DCA injection before the probe trial had no effect on memory performance in mice that had already undergone regular training, indicating that $A G$ is not explicitly required for memory retrieval. In light of these observations, we theorize that the transient increase in AG is required for the initial phases of learning, but not retrieval of established memories.

In support of this theory, several studies (Fox et al., 1988; Madsen et al., 1998, 1999) have shown that AG is quickly upregulated in response to neural stimulation and can account for up to $40 \%$ of the glucose consumed by active brain regions. In a recent study (Shannon et al., 2016), PET scans of humans before and after a visualmotor adaptation task revealed that the left Brodmann area 44 played a key role in task performance that correlated strongly with an increase in AG in the same brain region. Thus, elevated $A G$ levels may arise to support the increased energetic demand in brain regions engaged in specific tasks. Moreover, AG plays a critical role in synaptic plasticity by enabling the synthesis of biosynthetic building blocks derived from glycolytic intermediates to support processes such as cytoskeletal rearrangements, lipid synthesis, protein trafficking, and increased gene expression (Yang et al., 2009; Bauernfeind et al., 2014; Bas-Orth et al., 2017). The spatial distribution of AG in the human brain also correlates with the expression of developmental and neotenous genes, strongly implicating AG in supporting new synapse formation and growth (Goyal et al., 2014). Indeed, recent studies (Yang et al., 2014; 
Margineanu et al., 2018) have shown that the exposure of mouse primary cortical neurons to lactate stimulates the expression of synaptic plasticity-related genes including Arc, c-Fos, Bdnf, and Egr1 through an NMDA receptordependent mechanism.

Whether AG is upregulated directly in astrocytes, neurons, or both is currently a contentious issue (Dienel, 2012). Uptake of the glucose analog 6-deoxy- $N-(7-$ nitrobenz-2-oxa-1,3-diazol-4-yl)-aminoglucose (NBDG), as assessed by in vivo two-photon imaging, revealed preferential uptake in astrocytes during activation of the somatosensory cortex in mice (Chuquet et al., 2010). Two-photon imaging of mouse hippocampal and cerebellar brain slices demonstrated that the transport and metabolism of the fluorescent glucose analog 2-NBDG is higher in astrocytes than in neurons located in their vicinity (Jakoby et al., 2014). In addition, confocal imaging of mouse primary cultures transfected with a genetically encoded FRET glucose biosensor revealed that glucose uptake is faster in astrocytes than neurons (Jakoby et al., 2014). However, several recent reports have provided evidence suggesting that glucose may also be taken up by neurons. Two-photon imaging analysis, using a nearinfrared fluorophore, IRDye $800 \mathrm{CW}$, conjugated to 2-deoxyglucose (2DG)-IR, revealed that 2DG-IR was taken up preferentially by neurons in awake behaving mice and that sensory stimulation leads to a pronounced increase in neuronal, but not astrocytic, 2DG-IR uptake (Lundgaard et al., 2015). However, it should be noted that 2DG-IR is likely internalized as a macromolecular complex with the glucose transporter Glu transporter (GluT) 1 by endocytosis and may not adequately reflect physiologic glucose uptake (Kovar et al., 2009). A separate study, using metabolic biosensors in hippocampal slices and brains of awake mice, revealed that neuronal stimulation is dependent on increased glucose consumption directly by neurons, not astrocytic-derived lactate (Díaz-García et al., 2017). Intrahippocampal injection of indinavir, a selective chemical inhibitor of the neuronal GluT4, before IA training in rats resulted in impaired learning, whereas inhibition of glucose transport after training had no effect on memory retrieval (Pearson-Leary and McNay, 2016). The discrepancies between astrocytic and neuronal uptake of glucose in the aforementioned studies may be attributed to a number of factors, including regional variation in glucose metabolism, the task-dependent nature of glucose uptake, and limitations when using glucose analogues and biosensors to measure glucose uptake and processing. Regardless, we propose that the transient elevation of neuronal glycolysis may act as a "first responder" to meet the increased energy needs of stimulated neurons due to the fact that glycolysis can generate ATP at a much faster rate than mitochondrial oxidative phosphorylation, while lactate production may spur the expression of immediate early genes involved in LTP.

Recent studies have shown that the glycolytic enzymes PDK1 and LDHA are primarily expressed within neurons of the frontal cortex and hippocampus of wild-type and transgenic Alzheimer's disease mice (Harris et al., 2016; Zhang et al., 2018). Interestingly, it was recently shown that the induction of synaptic activity promotes increased neuronal glucose uptake and the expression of glycolytic genes (Bas-Orth et al., 2017; Segarra-Mondejar et al., 2018). Moreover, synaptic activity triggered increases in glucose metabolism and promotes the generation of lipid precursors required for cell membrane enlargement during neurite outgrowth (Segarra-Mondejar et al., 2018). It is possible that $A G$ occurs in neurons during the initial phases of learning to promote structural synaptic remodeling within memory engram neurons. Once memory acquisition and synaptic remodeling have occurred, then mitochondrial oxidative phosphorylation may play a prominent role in ensuring that sufficient ATP production occurs to maintain established connections associated with memory recall. It is our intention that these findings will spur future studies to further dissect apart the role of either astrocytic or neuronal $A G$ in various paradigms of learning and memory.

While our findings suggest that $A G$ is required for memory acquisition, we could not determine the role of $A G$ on memory consolidation due to the limitations of the experimental design used in this study. Memory consolidation has typically been evaluated using classical behavioral assays with rapid acquisition such as the passiveavoidance task (Lorenzini et al., 1996) or fear conditioning (Schafe et al., 1999). It is generally easier to differentiate post-training events, such as memory consolidation, from learning when the duration of the acquisition phase is short. The multiple training sessions used in the MWM over $4 \mathrm{~d}$ may result in memory traces that are reactivated and reconsolidated during each learning session, possibly obscuring events associated with the initial consolidation of a spatial memory (Florian and Roullet, 2004). However, several studies have made use of a massed training protocol for the MWM in which mice are submitted to four consecutive trial sessions within a 70-90 min period followed by a probe trial $24 \mathrm{~h}$ later (Florian and Roullet, 2004; Stein et al., 2014; Villain et al., 2016). The $1 \mathrm{~d}$ protocol can expose deficits in early consolidation after the training sessions without the complication of overlapping learning and consolidation processes during multiday training protocols (Stein et al., 2014). In future studies, it would be of interest to use the $1 \mathrm{~d}$ MWM training protocol and administer DCA after the condensed training sessions to determine whether the inhibition of $A G$ also interferes with the early events associated with memory consolidation.

\section{References}

Abemayor E, Kovachich GB, Haugaard N (1984) Effects of dichloroacetate on brain pyruvate dehydrogenase. J Neurochem 42:3842. CrossRef Medline

Bas-Orth C, Tan Y-W, Lau D, Bading H (2017) Synaptic activity drives a genomic program that promotes a neuronal Warburg effect. J Biol Chem 292:5183-5194. CrossRef Medline

Bauernfeind AL, Barks SK, Duka T, Grossman LI, Hof PR, Sherwood CC (2014) Aerobic glycolysis in the primate brain: reconsidering the implications for growth and maintenance. Brain Struct Funct 219:1149-1167. CrossRef Medline

Boyle PJ, Scott JC, Krentz AJ, Nagy RJ, Comstock E, Hoffman C (1994) Diminished brain glucose metabolism is a significant determinant for falling rates of systemic glucose utilization during sleep in normal humans. J Clin Invest 93:529-535. CrossRef Medline 
Brown AM, Baltan Tekkök S, Ransom BR (2004) Energy transfer from astrocytes to axons: the role of CNS glycogen. Neurochem Int 45:529-536. CrossRef Medline

Chuquet J, Quilichini P, Nimchinsky EA, Buzsáki G (2010) Predominant enhancement of glucose uptake in astrocytes versus neurons during activation of the somatosensory cortex. J Neurosci 30:15298-15303. CrossRef Medline

Díaz-García CM, Mongeon R, Lahmann C, Koveal D, Zucker H, Yellen G (2017) Neuronal Stimulation Triggers Neuronal Glycolysis and Not Lactate Uptake. Cell Metab 26:361-374.e4. CrossRef Medline

Dienel GA (2012) Brain lactate metabolism: the discoveries and the controversies. J Cereb Blood Flow Metab 32:1107-1138. CrossRef Medline

Florian C, Roullet P (2004) Hippocampal CA3-region is crucial for acquisition and memory consolidation in Morris water maze task in mice. Behav Brain Res 154:365-374. CrossRef Medline

Fox PT, Raichle ME, Mintun MA, Dence C (1988) Nonoxidative glucose consumption during focal physiologic neural activity. Science 241:462-464. CrossRef Medline

Goyal MS, Hawrylycz M, Miller JA, Snyder AZ, Raichle ME (2014) Aerobic glycolysis in the human brain is associated with development and neotenous gene expression. Cell Metab 19:49-57. CrossRef Medline

Harris RA, Tindale L, Lone A, Singh O, Macauley SL, Stanley M, Holtzman DM, Bartha R, Cumming RC (2016) Aerobic glycolysis in the frontal cortex correlates with memory performance in wild-type mice but not the APP/PS1 mouse model of cerebral amyloidosis. J Neurosci 36:1871-1878. CrossRef Medline

Jakoby P, Schmidt E, Ruminot I, Gutiérrez R, Barros LF, Deitmer JW (2014) Higher transport and metabolism of glucose in astrocytes compared with neurons: a multiphoton study of hippocampal and cerebellar tissue slices. Cereb Cortex 24:222-231. CrossRef Medline

Kato M, Wynn RM, Chuang JL, Tso S-C, Machius M, Li J, Chuang DT (2008) Structural basis for inactivation of the human pyruvate dehydrogenase complex by phosphorylation: role of disordered phosphorylation loops. Structure 16:1849-1859. CrossRef Medline

Kovar JL, Volcheck W, Sevick-Muraca E, Simpson MA, Olive DM (2009) Characterization and performance of a near infrared 2-deoxyglucose optical imaging agent for mouse cancer models. Anal Biochem 384:254-262. CrossRef Medline

Lorenzini CA, Baldi E, Bucherelli C, Sacchetti B, Tassoni G (1996) Role of dorsal hippocampus in acquisition, consolidation and retrieval of rat's passive avoidance response: a tetrodotoxin functional inactivation study. Brain Res 730:32-39. CrossRef Medline

Lundgaard I, Li B, Xie L, Kang H, Sanggaard S, Haswell JDR, Sun W, Goldman S, Blekot S, Nielsen M, Takano T, Deane R, Nedergaard M (2015) Direct neuronal glucose uptake heralds activitydependent increases in cerebral metabolism. Nat Commun 6:6807. CrossRef Medline

Madsen PL, Linde R, Hasselbalch SG, Paulson OB, Lassen NA (1998) Activation-induced resetting of cerebral oxygen and glucose uptake in the rat. J Cereb Blood Flow Metab 18:742-748. CrossRef Medline

Madsen PL, Cruz NF, Sokoloff L, Dienel GA (1999) Cerebral oxygen/ glucose ratio is low during sensory stimulation and rises above normal during recovery: excess glucose consumption during stimulation is not accounted for by lactate efflux from or accumulation in brain tissue. J Cereb Blood Flow Metab 19:393-400. CrossRef Medline

Magistretti PJ, Allaman I (2018) Lactate in the brain: from metabolic end-product to signalling molecule. Nat Rev Neurosci 19:235-249. CrossRef Medline

Margineanu MB, Mahmood H, Fiumelli H, Magistretti PJ (2018) $\mathrm{L}$-Lactate regulates the expression of synaptic plasticity and neuroprotection genes in cortical neurons: a transcriptome analysis. Front Mol Neurosci 11:375. CrossRef Medline

McNay EC, Fries TM, Gold PE (2000) Decreases in rat extracellular hippocampal glucose concentration associated with cognitive de- mand during a spatial task. Proc Natl Acad Sci U S A 97:28812885. CrossRef Medline

McNay EC, Williamson A, McCrimmon RJ, Sherwin RS (2006) Cognitive and neural hippocampal effects of long-term moderate recurrent hypoglycemia. Diabetes 55:1088-1095. CrossRef Medline

Mergenthaler P, Lindauer U, Dienel GA, Meisel A (2013) Sugar for the brain: the role of glucose in physiological and pathological brain function. Trends Neurosci 36:587-597. CrossRef Medline

Newman LA, Korol DL, Gold PE (2011) Lactate produced by glycogenolysis in astrocytes regulates memory processing. PLoS One 6:e28427. CrossRef Medline

Newman LA, Scavuzzo CJ, Gold PE, Korol DL (2017) Traininginduced elevations in extracellular lactate in hippocampus and striatum: dissociations by cognitive strategy and type of reward. Neurobiol Learn Mem 137:142-153. CrossRef Medline

Pearson-Leary J, McNay EC (2016) Novel roles for the insulinregulated glucose transporter-4 in hippocampally dependent memory. J Neurosci 36:11851-11864. CrossRef Medline

Powers WJ, Videen TO, Markham J, McGee-Minnich L, AntenorDorsey JV, Hershey T, Perlmutter JS (2007) Selective defect of in vivo glycolysis in early Huntington's disease striatum. Proc Natl Acad Sci U S A 104:2945-2949. CrossRef Medline

Raichle ME, Posner JB, Plum F (1970) Cerebral blood flow during and after hyperventilation. Arch Neurol 23:394-403. CrossRef Medline

Sandusky LA, Flint RW, McNay EC (2013) Elevated glucose metabolism in the amygdala during an inhibitory avoidance task. Behav Brain Res 245:83-87. CrossRef Medline

Schafe GE, Nadel NV, Sullivan GM, Harris A, LeDoux JE (1999) Memory consolidation for contextual and auditory fear conditioning is dependent on protein synthesis, PKA, and MAP kinase. Learn Mem 6:97-110. Medline

Segarra-Mondejar M, Casellas-Díaz S, Ramiro-Pareta M, MüllerSánchez C, Martorell-Riera A, Hermelo I, Reina M, Aragonés J, Martínez-Estrada OM, Soriano FX (2018) Synaptic activity-induced glycolysis facilitates membrane lipid provision and neurite outgrowth. EMBO J 37:e97368. CrossRef Medline

Shannon BJ, Vaishnavi SN, Vlassenko AG, Shimony JS, Rutlin J, Raichle ME (2016) Brain aerobic glycolysis and motor adaptation learning. Proc Natl Acad Sci U S A 113:E3782-E3791. CrossRef Medline

Stacpoole PW, Nagaraja NV, Hutson AD (2003) Efficacy of dichloroacetate as a lactate-lowering drug. J Clin Pharmacol 43:683-691. CrossRef Medline

Stein IS, Donaldson MS, Hell JW (2014) CaMKII binding to GluN2B is important for massed spatial learning in the Morris water maze. F1000Res 3:193. CrossRef Medline

Suzuki A, Stern SA, Bozdagi O, Huntley GW, Walker RH, Magistretti $\mathrm{PJ}$, Alberini CM (2011) Astrocyte-neuron lactate transport is required for long-term memory formation. Cell 144:810-823. CrossRef Medline

Tadi M, Allaman I, Lengacher S, Grenningloh G, Magistretti PJ (2015) Learning-induced gene expression in the hippocampus reveals a role of neuron-astrocyte metabolic coupling in long term memory. PLoS One 10:e0141568. CrossRef Medline

Villain H, Florian C, Roullet P (2016) HDAC inhibition promotes both initial consolidation and reconsolidation of spatial memory in mice. Sci Rep 6:27015. CrossRef Medline

Yang G, Pan F, Gan W-B (2009) Stably maintained dendritic spines are associated with lifelong memories. Nature 462:920-924. CrossRef Medline

Yang J, Ruchti E, Petit J-M, Jourdain P, Grenningloh G, Allaman I, Magistretti PJ (2014) Lactate promotes plasticity gene expression by potentiating NMDA signaling in neurons. Proc Natl Acad Sci U S A 111:12228-12233. CrossRef Medline

Zhang M, Cheng X, Dang R, Zhang W, Zhang J, Yao Z (2018) Lactate deficit in an Alzheimer disease mouse model: the relationship with neuronal damage. J Neuropathol Exp Neurol 77:1163-1176. CrossRef Medline 